\title{
Nursing/Pharmacy
}

\section{FIGHTING THE WAR AGAINST COVID-19: ADMINISTRATION OF BAMLANIVIMAB (BAM) OR BAMLANIVIMAB + ETESIVIMAB (BAM + E); A COOPERATIVE EFFORT BETWEEN A COMMUNITY CANCER CENTER AND AN URGENT CARE (UC) FACILITY}

${ }^{1}$ Patrick Skeffington, ${ }^{2}$ Robert Aisenberg, ${ }^{1}$ Janice Dallacosta*, ${ }^{1}$ lan Donaghy, ${ }^{2}$ Dani Hackner, ${ }^{2}$ Kelly Houde, ${ }^{1}$ Kathy Moraes, ${ }^{1}$ Annemarie Santos. 'Southcoast Centers for Cancer Care, Fairhaven, MA, USA; ${ }^{2}$ Southcoast Health, Fall River, MA, USA

Background Goal of the Massachusetts DPH is to ensure equitable distribution of BAM to the most vulnerable at risk of poor outcomes from COVID-19 and to communities with the highest incidences of COVID-19. Hospitals should allocate available doses in a manner consistent with this guidance: 1 . Patients who meet the EUA criteria; a lottery system will be used if supply is exceeded 2. Patients with comorbidities (high risk) tend to have worse outcomes when infected with SARSCoV-2 3. BAM was approved under an EUA for the treatment of mild to moderate COVID-19 for those at high risk of progressing to severe disease (revoked 4/16/21). 4. BAM + E combo was approved under an EUA for the same patients and criteria, Southcoast Health entered into this relationship with $\mathrm{DPH}$ to provide this service to the southeastern MA population.

Methods Patients identified based on algorithm using Social Vulnerability Index (SVI) and EUA criteriaRNs screened cases for positive criteria using lottery priority and SVIPulmonologists consented appropriate patients, ordered infusions, routed cases for final scheduling within window of treatmentExperienced nursing staff from various Southcoast departments treated up to 6 patients per dayOncology pharmacies are uniquely experienced to prepare monoclonal antibodies (MABS) such as BAM and BAM + EDue to proximity of the Oncology pharmacy to the UC Center, pharmacy reviewed, prepared and delivered infusions to UC once patient was assessed by RNs

Results First 152 cases: 7.2\% inpatient admissions within 14 days $13.8 \% \mathrm{ED} / \mathrm{UC}$ visits within 14 days2\% inpatient admissions in 28 days5.9\% ED/UC visits within 28 daysTwo deaths during initial 152 cases.

Conclusions Cooperative effort between the Cancer Center and Urgent Care led to positive outcomes for local COVID19 patients. Southcoast demonstrated a $6 \%$ hospital admission rate for COVID-19 patients in the MAB program versus $26 \%$ admission rate overall for COVID-19 patients.

Acknowledgements Thanks to our colleagues at the University of Rhode Island College of Pharmacy for their support with the poster

http://dx.doi.org/10.1136/jitc-2021-SITC2021.896 